\title{
Pseudomonas Keratitis, a Review of Where We’ve Been and What Lies Ahead
}

\author{
Alicia Eby $\mathbf{M}^{1}$ and Linda Hazlett $\mathrm{D}^{2 *}$
}

${ }^{1}$ Kresge Eye Institute, Wayne State University, Detroit Michigan, United States

${ }^{2}$ Department of Anatomy and Cell Biology, Wayne State University School of Medicine, Detroit Michigan, United States

\begin{abstract}
Microbial keratitis can result in significant vision loss secondary to corneal scarring or surface irregularity. If untreated, corneal perforation and endophthalmitis can occur, resulting in loss of the eye. Damage to the cornea can occur in as little as $24 \mathrm{hrs}$ and the most aggressive infections are often caused by $P$. aeruginosa which is a highly virulent opportunistic pathogen. This bacterium is highly effective at evading the immune response and blunting it via several mechanisms. Normally the innate immune system provides defense with neutrophils which are recruited within as little as 6-12 hrs of infection travelling from limbal vessels via the tear film to the site of the infection. These cells play a critical role in limiting bacterial proliferation as well as protecting host tissue from destruction, but if they persist locally they are detrimental, inducing additional tissue damage. Evasion is aided in part due to the bacterial glycocalyx which functions to inhibit migration and function of neutrophils. Beyond this first line of defense, the complex story unravels involving secreted exotoxins $\mathrm{S}, \mathrm{T}$ and $\mathrm{U}$, the role of TLRs, chemokines and cytokines including interleukins 1, 6, 10,12, 17 and 18, IFN- $\gamma$ as well as CD4+ T cells, antigen presenting cells (Langerhans cells) and macrophages. With a complex interplay between bacteria and host, infection with $P$. aeruginosa has a hallmark ability to incite an exuberant immune response, propagating local tissue damage. It is facile to appreciate just how difficult it has been to target precise susceptibilities, treatment strategies and models of pathogenesis, given the elaborate mechanisms involved in both bacterial action and more importantly host inflammation. Herein we will review some of the major breakthroughs in our understanding of the pathogenesis of this bacterium and discuss recent novel therapeutic targets.
\end{abstract}

Keywords: Pseudomonas; Keratitis; Corneal ulcer

\section{Background}

Pseudomonas aeruginosa ( $P$. aeruginosa) is a gram negative bacterium that often contaminates water and is a formidable opportunistic pathogen responsible for some of the most severe infections of the eye, including bacterial keratitis and endophthalmitis [1]. Contact-lens related infectious keratitis is the most common presentation of ocular $P$. aeruginosa infection. Soft, extendedwear contact lenses are the most common culprit. Visual loss is a consequence of subsequent scarring of the cornea and in fulminant cases can lead to perforation or extension into surrounding tissues. Several factors mediate the virulence of this organism including flagella, adhesins, toxin secretion, proteases and pili [2]. Our knowledge of the pathogenesis of pseudomonal ulcers is largely owed to animal models, particularly rodents, and now an extended wear contact lens fitted in the rat allows simulation of the natural evolution of the disease [3].

The investigations into bacterial keratitis have brought to light that although exoenzymes secreted by pathogenic bacteria initiate the insult to the cornea, much of the degradation of host tissue occurs as a consequence of uncontrolled inflammation with release of potent immunological mediators from corneal cells and infiltrating white blood cells, predominately neutrophilic (PMN) leukocytes $[4,5]$.

\section{Epidemiology in Contact Lens Wear}

Up to one-third of emergency room visits for corneal infection are contact lens wear-related corneal infection as contact lens wear imparts a tenfold increase in the risk for corneal infection. As much as 1942 percent of bacterial keratitis is related to contact lens usage. The risk increases fifteen-fold for those who sleep in their contact lenses [2]. Even with the advent of silicone hydrogel lenses with high DK/t oxygen transmissibility, the rate of bacterial keratitis appears to have remained unchanged [6]. There are now an estimated 38 million contact lens wearers in the US. Annual incidence of microbial keratitis continues to rise and now accounts for 930,000 outpatient visits and 58,000 emergency department visits per year [7]. The immunocompromised, debilitated and hospitalized as well as those in tropical climates are also susceptible to pseudomonal infections [8]. This imparts a significant burden on the healthcare system with a financial cost of approximately 175 million USD/year [7]. There are other important factors in addition to contact lens wear that also can result in eye infections with this pathogen, including agricultural accidents, but they are not the focus of this review.

\section{Diagnosis}

A careful medical history will often reveal contact lens wear. For microbial keratitis, general presentation consists of symptoms including photophobia, decreased vision and pain. Clinical findings may include conjunctival injection, single focal ulcer with welldelineated loss of epithelium associated with an ill-defined suppurative stromal infiltrate. There is often accompanying corneal edema, mucopurulent exudate and anterior chamber reaction with possible

*Corresponding author: Linda Hazlett D, Department of Anatomy and Cell Biology, Wayne State University School of Medicine, Detroit Michigan, United States, Tel: +1-313-577-1079; E-mail: Ihazlett@med.wayne.edu

Received December 03, 2015; Accepted December 14, 2015; Published December 21, 2015

Citation: Alicia Eby M, Linda Hazlett D (2016) Pseudomonas Keratitis, a Review of Where We've Been and What Lies Ahead. J Microb Biochem Technol 8: 009-013. doi:10.4172/1948-5948.1000254

Copyright: (c) 2016 Alicia Eby M, et al. This is an open-access article distributed under the terms of the Creative Commons Attribution License, which permits unrestricted use, distribution, and reproduction in any medium, provided the original author and source are credited. 
hypopyon formation. However, presentation alone is not predictive of a specific microbial pathogen, thus corneal scraping and culture should be performed prior to initiation of empiric broad-spectrum antibiotic therapy [2]. In the case of $P$. aeruginosa, disease presentation often consists of a corneal ulcer paracentrally located with a ring of infiltrate and yellow coagulative necrosis with stromal ulceration, epithelial edema and mucopurulent exudate in addition to the general symptoms described above [9].

\section{Pathogenesis}

Contact lenses increase the likelihood of corneal infection due to several factors including extended exposure of the eye to contamination via the foreign body, disturbance to the natural tear film, microtrauma to the corneal surface epithelium, perturbation of the immune function at the surface of the eye, hypoxic status of the cornea in the setting of contact lens wear and improper lens or eye hygiene leading to inoculation of the ocular surface [2]. The eye is also predisposed to infection with $P$. aeruginosa in the setting of other ocular pathology including Herpes simplex infection, immunocompromise and ocular trauma [1]. Once the eye is inoculated with $P$. aeruginosa, there is adherence to the epithelium, followed by invasion into the corneal stroma and subsequent proliferation which is often exaggerated by the host immune reaction. It has been suggested that adherence of the organism to epithelium can occur within 15 minutes and bacteria reach the stroma in as little as 60 minutes [10].

Adherence- The tear layer overlying the cornea plays an important role in the defense against microorganisms. It not only provides lubrication and a physical barrier with layers composed of lipid, mucin, and aqueous, but contains factors involved in healing including epidermal growth factor. Lactoferrin, defensins, and IgA are also found in the tear film to protect against bacterial invasion. Interleukin-1 receptor antagonist (IL-1RA), transforming growth factor $\beta$ (TGF- $\beta$ ) and tissue inhibitor of matrix metalloproteinase 1 (TIMP-1) all play a role in protecting the cornea from uncontrolled and unregulated inflammation [11]. As demonstrated by Dart and Seal in the rabbit model, $P$. aeruginosa was undetectable in the cornea within $4 \mathrm{hrs}$ of inoculation unless trauma was induced [1]. Hypoxia is appreciated as a major predisposing factor for the bacteria to invade the cornea. It is thus important to recognize that PMNs are equipped to function under very low $\mathrm{O} 2$ conditions still using the glycolytic pathway with the assistance of hypoxia-inducible factors (HIF-1 $\alpha$ ) [12]. HIF-1 $\alpha$ is a transcription factor that has been studied in $\mathrm{BALB} / \mathrm{c}$ mice which resist infection (cornea does not perforate) but when this factor is suppressed using siRNA and/or antagonist 17-DMAG, the resistant phenotype is lost. There was no change in the number of PMNs or cytokines present, however there was a downstream effect whereas nitric oxide (NO) production was compromised, as well as bacterial killing and host cell apoptosis [13]. Zaidi et al. [14] investigated the role of cystic fibrosis transmembrane conductance regulator (CFTR) expression in rabbit and human corneas subjected to hypoxia for 24-72 hrs and found CFTR had increased expression as well as NF- $\kappa \mathrm{B}$ levels, which augmented the ability of $P$. aeruginosa to bind to epithelial cells, penetrate the cornea and induce a local inflammatory reaction.

In the setting of trauma, adherence is mediated by surface pili and glycocalyx allowing attachment to the epithelium. $P$. aeruginosa glycocalyx has been found to be immunogenic but not directly toxic to the cornea [1]. This extra-cellular mix of lipopolysaccharide and glycolipoprotein functions to inhibit migration and endocytosis of PMNs [13].
In vitro studies demonstrate that epithelial cells from corneas when exposed to bacterial factors isolated from extended wear contact lenses for at least $72 \mathrm{hrs}$, have impaired expression of antimicrobial peptide human $\beta$-defensin -2 (hBD-2) and surface protein D [15,16]. Defensins have been studied in murine models of the disease and found to be present in the normal cornea of both BALB/c and C57BL/6 (B6) mice. Once the corneas of these mice were inoculated with $P$. aeruginosa, there was a marked upregulation of murine $\beta$-defensins (mBD) 1 and 2 in the $\mathrm{BALB} / \mathrm{c}$ resistant mice. To evaluate the significance of this finding, siRNA was administered to knockdown $\mathrm{mDB} 1$ and 2. The silencing of $\mathrm{mBD} 2$ in $\mathrm{BALB} / \mathrm{c}$ mice resulted in increased disease severity and corneal damage, however no significant effect was found when interfering with mBD1 [17]. The mouse homologs of human (h) BD2 and 3 are $\mathrm{mBD} 3$ and 4 and their function was also studied in the mouse model of $P$. aeruginosa keratitis. It was found that mBD3 but not 4 was integral in host defense in BALB/c resistant vs B6 susceptible (cornea perforates after infection) mice. Not only was $\mathrm{mBD} 3$ upregulated in $\mathrm{BALB} / \mathrm{c}$ mice but it interacts with $\mathrm{mBD} 2$ to augment host defense against infection. Using siRNA, mBD4 knockdown had no appreciable effect; however $\mathrm{mBD} 3$ silencing revealed worsened corneal disease [18]. These defensins and their disparate expression in the two mouse strains may be, at least in part, the etiology for resistant vs susceptible mouse phenotypes.

Invasion- This process involves microbial proteases with keratolytic action leading to liquefactive necrosis of epithelial, stromal and endothelial cells, exotoxin $\mathrm{A}$ and $\mathrm{S}$ that function to prevent host protein synthesis, and leukocidin and haemolysins which create the environment for infiltration of bacteria. More specifically, leukocidin is a protein secreted by the organism which imparts toxicity to PMNs altering membrane permeability, permitting calcium entry and fusion of nuclear components [19]. These releasable exotoxins with a type III secretion system are especially capable of causing tissue necrosis in the cornea due to the avascular nature of the tissue which delays delivery of first-line host response cells such as PMNs [1]. Type III secretion delivers ExoS, ExoT and ExoU exotoxins into the cytosol and functions to prevent attack of bacteria as well as to promote PMN death [20]. Invasive strains typically express the exoS gene and are known to readily invade epithelial cells whereas cytotoxic strains express the exoU gene and release a phospholipase that induces host tissue necrosis in 1-2 hrs [21]. Contact lens wearers tend to be infected with the cytotoxic and/or invasive strains of $P$. aeruginosa [22]. Exotoxin A, found in 90 percent of $P$. aeruginosa species, is released by the organism and functions to inhibit host protein synthesis via ADP-ribosylation of elongation factor 2 [23]. Neutral protease, or elastase, is destructive to elastin although not native to the cornea; it also acts on proteoglycans which are present in corneal stroma in two types: decorin and lumican. The destruction of proteoglycans causes separation of collagen fibrils and loss of integrity of the normal compact lamellae of the cornea [24]. This loss of architecture and inflammation in the cornea leads to corneal edema and significant decreases in visual acuity as well as increased discomfort in the patient. Exotoxin $\mathrm{T}$ also predominates in pseudomonal corneal ulcers, co- expressed with exo $\mathrm{S}$ and functions to facilitate PMN death, knocking out an important component of the first line of host defense [25]. ExoU is expressed in only 10 percent of isolates and is a phospholipase capable of inducing inflammation and causing degradation of tissue $[20,26]$. These strains are identified as cytotoxic strains with the primary effect being cell lysis, whereas ExoS/T strains are non-cytotoxic and are functionally invasive via alteration of epithelial cell membranes and replication within these surface cells. The cytotoxic phenotypes tend to cause more aggressive infection and severe disease $[27,28]$. 
Host Inflammation- Release of host cytokines and chemokines recruiting host inflammatory cells are delivered to the avascular cornea via the tear layer and conjunctival blood vessels at the corneal limbus. Leukocytic cells release matrix metalloproteinases precipitating necrosis of the cornea tissue [2]. PMNs are critical for the elimination of pathogens especially early in infection. However phagocytosis has unwanted secondary consequences due to degranulation of PMNs that lead to innocent bystander damage, including destruction of epithelium and stroma leading to corneal necrosis [29]. PMNs have also been implicated in the scarring and perforation that can occur with pseudomonal infection [30]. Immune recognition of the bacterium in macrophages is mediated by the IPAF/NLRC4 inflammasome [31] but in keratitis (murine) PMN serine proteases were found of importance [32]. Murine models of pseudomonal keratitis have been long established and continue to provide valuable insight into the complex interaction between direct toxicity of $P$. aeruginosa on the cornea and local host factors that collectively contribute to tissue destruction. Macrophage inflammatory protein-2 (MIP-2) and KC in the mouse are chemokines analogous to interleukin 8 (IL-8) and GROa in humans which function to recruit and activate PMNs. MIP-2, in particular is found at increased concentrations in B6 "susceptible" mouse models of $P$. aeruginosa keratitis whose infected cornea perforates [33]. IL$1 \beta$ is a cytokine produced by macrophages, monocytes and corneal cells alike upon infection, and has been found to contribute to PMN recruitment. Higher levels of IL-1 $\beta$ are detected in B6 mice but much lower levels are seen in BALB/c "resistant" mice which tend to avoid progression to perforation. Inhibition of IL- $1 \beta$ in B6 mice resulted in reduction in the severity of disease, decreased presence of PMNs in the cornea, decreased pathogen load, and concentration of localMIP-2 [34]. Macrophages themselves were studied in this murine model by cell depletion prior to inoculation with $P$. aeruginosa. B6 susceptible mice and $\mathrm{BALB} / \mathrm{c}$ resistant mice both had earlier onset and more severe disease states whereas B6 corneas perforated at day 3 vs 5-7 days and $\mathrm{BALB} / \mathrm{c}$ perforated at 5-7 days which is unlike their normal resistance state [30]. T cells, particularly CD4+ cells, have also been implicated in corneal $P$. aeruginosa infection and have been studied in murine models. B6 wild type mice infected with $P$. aeruginosa underwent perforation within 7 days however when these mice are depleted of their CD4+ cells (and IFN- $\gamma$ levels) they failed to progress to perforation unlike CD8+ depleted animals which retained their tendency to perforation [35]. CD4+ T cells, specifically Th17 cells, have recently been studied for their role in the immunopathology of $P$. aeruginosa infection. With the use of galectin-1 (Gal-1) to bind carbohydrates on CD4+ cells, a significant diminution in disease severity was observed in the infected corneas of $\mathrm{B} 6$ mice. There was a marked decreased in the number of PMNs, CD4+ and CD45+ cells, as well as a decreased presence of Th17+ cells in infected tissue [36]. This work suggests that Th17 cells, which secrete IL-17, a proinflammatory cytokine, drive a significant portion of the exuberant host response in pseudomonal ulcers and treatment aimed at knocking down Th17 cells may provide a future therapeutic target.

In the setting of contact lens wear, Langerhans cells (LC) have been found to migrate from their residence in the limbal conjunctiva into the cornea. When $\mathrm{BALB} / \mathrm{c}$ resistant mice were infected with $P$. aeruginosa following induction of LC migration (using sterile bead application) into the cornea before infection, increased levels of LCs and their expression of B7- 1 were detected and the tendency towards corneal perforation was increased. Also, when B6 susceptible mice were given anti-B7 antibodies, there was a reduction in disease severity and fewer CD4+ T cells present [30]. This suggests that LCs have a proinflammatory role that can exaggerate the host reaction and be ultimately detrimental to the cornea in P. aeruginosa keratitis.

There is evidence that Toll-like receptors (TLR) play a role in the immune response in $P$. aeruginosa keratitis whereas there is increased expression of TLRs in susceptible compared with resistant mice [37]. In humans TLR5 specifically is detected in corneal epithelium of humans and functions to bind bacterial flagellin which then initiates a signaling cascade to augment inflammation [30]. TLRs in the mouse have been found to be activated by mBD-2 first via its action on dendritic cells which then functions as a ligand for TLR4 and stimulation of the adaptive immune response [38]. In human corneas CD14 and TLR4 are expressed and upon interaction with lipopolysaccharide (LPS) there is a calcium dependent process that occurs releasing a variety of cytokines and chemokines and propagating the immune response [39]. It is an area requiring further investigation but has promise for unlocking more clues to the pathogenesis of bacterial keratitis.

\section{Current Prevention and Treatment}

Proper lens hygiene including using fresh solution each day, replacing lens cases, hand washing, avoiding sitting water in the eye and wearing well-fitting contacts are all important factors in preventing the development of bacterial keratitis associated with contact-lens wear. Daily disposable lenses appear more favorable to be recommended to patients as they impart the lowest risk of infection [40]. Avoidance of any patching of the eye in the setting of contact-lens related corneal infection is paramount in preventing progression of disease. Prompt initiation of broad antibiotic coverage is critical as corneal infections due to $P$. aeruginosa can progress precipitously. Corticosteroid administration should be avoided prior to initiation of antibiotic therapy. The use of corticosteroids with concurrent antibiotic therapy remains controversial. A large multicenter randomized clinical trial, The Steroids for Corneal Ulcers Trial (SCUT) was conducted to evaluate the therapeutic role of steroid use in the form of prednisolone sodium phosphate 1.0 percent as an adjunct to antibiotic therapy in bacterial keratitis and found there was overall no difference in the best corrected visual acuity at 3 months however there were also no safety concerns associated with its use. However, in patients with counting fingers or worse vision and those with central ulcers had a significant improvement in visual acuity with steroid administration [41].

Fluoroquinolones offer highly effective corneal penetration and coverage for pseudomonas species but for gram positive coverage including MRSA often combination therapy with vancomycin is warranted. Other options for the treatment of pseudomonal keratitis include tobramycin and ceftazidime. Fortified highconcentration formulations may be considered as well with caveat of toxicity to the corneal epithelium as well as cost and availability. When adjacent tissues are involved systemic antibiotics may be indicated. Once culture results are available revealing bacterial species and sensitivities, or if clinical response changes, treatment can be narrowed with monotherapy. In the setting of corneal perforation or descemetocele, a penetrating keratoplasty may be indicated [2].

\section{Current and Future Investigations}

Translating current basic research into a treatment setting is the ultimate goal of such studies, and as far as the role of modulating hostspecific factors, it would appear efficacious to hasten bacterial killing, and suppress the local exuberant and unregulated immune response. This is a lofty goal, given the superb complexity of the innate immune response to $P$. aeruginosa infections in the eye. 
One promising therapy involves suppression of highmobility group box 1 (HMGB1) an alarmin, and a member of a family of danger associated molecular patterns (DAMPS) that amplifies inflammation. In the susceptible B6 murine model, HMGB1 knockdown with siHMGB1 decreased corneal disease as well as diminished levels of IL- $1 \beta$, MIP-2, TNF- $\alpha$ and increased levels of antiinflammatory markers, and decreased numbers of infiltrated PMNs [42]. Investigations into this molecule are ongoing and may be a promising gateway into protection against bacterial keratitis using immunomodulatory chemical mediators or biologic pharmacotherapy using antibodies or other small molecules targeted to abrogate the effects of HMGB1 coupled with antibiotic therapy.

Another molecule being targeted for potential therapeutic value is Resolvin E1 (RvE1) which functions to recruit monocytes and macrophages which are non-inflammatory as well as encourage removal of PMNs from the site of infection [43]. Using a murine model, one group demonstrated that RvE1 treated corneas dampened the corneal inflammatory response initiated by LPS, $P$. aeruginosa and Staphylococcus aureus alike. There were decreased levels of cytokines, fewer macrophages and PMNs, and blunted formation of corneal infiltrates [44]. This suggests a potentially valuable role in the treatment of bacterial keratitis likely concomitant with antibiotics.

Given the severity of pseudomonal ulcers and their tendency to progress to perforation, it raises the question of the role of strengthening the corneal integrity using a technique with excellent efficacy in keratoconus. Iseli et al. [45] investigated the use of ultraviolet $\mathrm{A}$ and riboflavin corneal cross-linking in patients with infectious keratitis with evidence of corneal melting. Five patients were treated with cross-linking therapy after it was clear their infection was refractory to systemic and topical antibiotic therapy. Cessation of corneal melting was appreciated in all eyes that underwent crosslinking and this treatment may play an important role in protecting eyes from progression of the disease as well as avoiding more invasive procedures such as keratoplasty.

With the risk of severe disease and burden to the patient and healthcare system with contact lens related microbial keratitis, questions arise regarding the utility of antimicrobial lenses or lens cases. The goal would be to prevent or disrupt the biofilm that is accumulated on the posterior lens surface. Zhu et al. [46] have been investigating lenses coated with fimbrolide as a potential inhibitor of bacterial growth, but it is yet to be determined how this may translate to clinical practice. Unfortunately, the former does not address the compliance related risks of extended contact lens wear including sleeping in lenses for multiple sequential nights and improper lens hygiene. All of these also carry the risk of selecting for resistant species of organisms, which introduces increasingly difficult challenges for treatment.

\section{References}

1. Dart JK, Seal DV (1988) Pathogenesis and therapy of Pseudomonas aeruginosa keratitis. Eye (Lond) 2 Suppl: S46-S55.

2. Laibson (2014) In Basic and Clinical Science Course. Microbiol 12: 83-154.

3. Szliter EA, Barrett RP, Gabriel MM, Zhang Y, Hazlett LD (2006) Pseudomonas aeruginosa-induced inflammation in the rat extended-wear contact lens model. Eye Contact Lens 32: 12-18.

4. Lausch RN, Chen SH, Tumpey TM, Su YH, Oakes JE (1996) Early cytokine synthesis in the excised mouse cornea. J Interferon Cytokine Res 16: 35-40.

5. Hazlett LD, Kreindler FB, Berk RS, Barrett R (1990) Aging alters the phagocytic capability of inflammatory cells induced into cornea. Curr Eye Res 9: 129-138.

6. Stapleton F, Keay L, Edwards K, Naduvilath T, Dart JK, et al. (2008) The incidence of contact lens-related microbial keratitis in Australia. Ophthalmology 115: $1655-1662$

7. Collier SA, Gronostaj MP, MacGurn AK, Cope JR, Awsumb KL, et al. (2014) Estimated burden of keratitis--United States, 2010. MMWR Morb Mortal Wkly Rep 63: 1027-1030.

8. Laibson PR (1990) Pseudomonas aeruginosa. In: Fraunfelder FT, Roya FH, editors. Current Ocular Therapy, The WB Saunders Co, Philadelphia.

9. Wilhelmus KR (1996) Bacterial keratitis. In: Pepose JS, Holland GN, Wilhelmus KR, editors. Ocular Infection and immunity. St Louis (MO): Mosby 970-1031.

10. Stern GA (1990) Pseudomonas keratitis and contact lens wear: the lens/eye is at fault. Cornea 9 Suppl 1: S36-38.

11. Pflugfelder SC (2011) Tear dysfunction and the cornea: LXVIII Edward Jackson Memorial Lecture. Am J Ophthalmol 152: 900-909.

12. Imtiyaz HZ, Simon MC (2010) Hypoxia-inducible factors as essential regulators of inflammation. Curr Top Microbiol Immunol 345: 105-120.

13. Berger EA, McClellan SA, Vistisen KS, Hazlett LD (2013) HIF-1a is essential fo effective PMN bacterial killing, antimicrobial peptide production and apoptosis in Pseudomonas aeruginosa keratitis. PLoS Pathog 9: e1003457.

14. Zaidi T, Mowrey-McKee M, Pier GB (2004) Hypoxia increases corneal cell expression of CFTR leading to increased Pseudomonas aeruginosa binding, internalization, and initiation of inflammation. Invest Ophthalmol Vis Sci 45 4066-4074.

15. Laharrague PF, Corberand JX, Fillola G, Gleizes BJ, Fontanilles AM, et al. (1984) In vitro effect of the slime of Pseudomonas aeruginosa on the function of human polymorphonuclear neutrophils. Infect Immun 44: 760-762.

16. Fleiszig SM, Evans DJ (2010) Pathogenesis of contact lens-associated microbial keratitis. Optom Vis Sci 87: 225-232.

17. Wu M, McClellan SA, Barrett RP, Hazlett LD (2009) Defensin-2 promotes resistance against Infection with $P$. aeruginosa. J Immunol 182: 1609-1616.

18. Wu M, McClellan SA, Barrett RP, Zhang Y, Hazlett LD (2009) Beta-defensins 2 and 3 together promote resistance to Pseudomonas aeruginosa keratitis. J Immunol 183: 8054-8060.

19. Lory S, Tai PC (1985) Biochemical and genetic aspects of Pseudomonas aeruginosa virulence. Curr Top Microbiol Immunol 118: 53-69.

20. Karthikeyan RS, Priya JL, Leal SM Jr, Toska J, Rietsch A, et al. (2013) Host response and bacterial virulence factor expression in Pseudomonas aeruginosa and Streptococcus pneumoniae corneal ulcers. PLoS One 8: e64867.

21. Fleisig SM, Zaidi TS, Preston MJ, Grout M, Evans DG, et al. (1996) Relationship between cytotoxicity and corneal epithelial cell invasion by clinical isolates of Pseudomonas aeruginosa. Infect Immun 64: 579-586.

22. Shen EP, Tsay RY, Chia JS, Wu S, Lee JW, et al. (2012) The role of type III secretion system and lens material on adhesion of Pseudomonas aeruginosa to contact lenses. Invest Ophthalmol Vis Sci 53: 6416-6426.

23. Hedstrom RC, Funk CR, Kaper JB, Pavlovskis OR, Galloway DR (1986) Cloning of a gene involved in regulation of exotoxin A expression in Pseudomonas aeruginosa. Infect Immun 51: 37-42.

24. Kessler E, Blumberg S (1987) Specific inhibitors of Pseudomonas aeruginosa elastase as potential drugs for the treatment of Pseudomonas keratitis. Antibiot Chemother (1971) 39: 102-112.

25. Sun Y, Karmakar M, Taylor PR, Rietsch A, Pearlman E (2012) ExoS and ExoT ADP ribosyltransferase activities mediate Pseudomonas aeruginosa keratitis by promoting neutrophil apoptosis and bacterial survival. J Immunol 188: 18841895

26. Hauser AR (2009) The type III secretion system of Pseudomonas aeruginosa: infection by injection. Nat Rev Microbiol 7: 654-665.

27. Angus AA, Lee AA, Augustin DK, Lee EJ, Evans DJ, et al. (2008) Pseudomonas aeruginosa induces membrane blebs in epithelial cells, which are utilized as a niche for intracellular replication and motility. Infect Immun 76: 1992-2001.

28. Cole N, Willcox MD, Fleiszig SM, Stapleton F, Bao B, et al. (1998) Different strains of Pseudomonas aeruginosa isolated from ocular infections or inflammation display distinct corneal pathologies in an animal model. Curr Eye Res 17: 730-735. 
Citation: Alicia Eby M, Linda Hazlett D (2016) Pseudomonas Keratitis, a Review of Where We've Been and What Lies Ahead. J Microb Biochem Technol 8: 009-013. doi:10.4172/1948-5948.1000254

29. Hyndiuk RA (1981) Experimental Pseudomonas keratitis. Trans Am Ophthalmol Soc 79: $541-624$

30. Hazlett LD (2004) Corneal response to Pseudomonas aeruginosa infection. Prog Retin Eye Res 23: 1-30.

31. Sutterwala FS, Mijares LA, Li L, Ogura Y, Kazmierczak BI, et al. (2007) Immune recognition of Pseudomonas aeruginosa mediated by the IPAF/NLRC4 inflammasome. J Exp Med 204: 3235-3245.

32. Karmakar M, Sun Y, Hise AG, Rietsch A, Pearlman E (2012) Cutting edge: IL-1 $\beta$ processing during Pseudomonas aeruginosa infection is mediated by neutrophil serine proteases and is independent of NLRC4 and caspase-1. J Immunol 189: 4231-4235.

33. Kernacki KA, Barrett RP, Hobden JA, Hazlett LD (2000) Macrophage inflammatory protein-2 is a mediator of polymorphonuclear neutrophil influx in ocular bacterial infection. J Immunol 164: 1037-1045

34. Rudner XL, Kernacki KA, Barrett RP, Hazlett LD (2000) Prolonged elevation of IL-1 in Pseudomonas aeruginosa ocular infection regulates macrophageinflammatory protein-2production, polymorphonuclear neutrophil persistence, and corneal perforation. J Immunol 164: 6576-6582.

35. Kwon B, Hazlett LD (1997) Association of CD4+ T cell-dependent keratitis with genetic susceptibility to Pseudomonas aeruginosa ocular infection. J Immunol 159: 6283-6290.

36. Suryawanshi A, Cao Z, Thitiprasert T, Zaidi TS, Panjwani N (2013) Galectin1-mediated suppression of Pseudomonas aeruginosa-induced corneal immunopathology. J Immunol 190: 6397-6409.

37. Huang X, Barrett RP, McClellan SA, Hazlett LD (2005) Silencing Toll-like receptor-9 in Pseudomonas aeruginosa keratitis. Invest Ophthalmol Vis Sci 46: 4209-4216.
38. Biragyn A, Ruffini PA, Leifer CA, Klyushnenkova E, Shakhov A, et al. (2002) Toll-like receptor 4-dependent activation of dendritic cells by beta-defensin 2 . Science 298: 1025-1029.

39. Song PI, Abraham TA, Park Y, Zivony AS, Harten B, et al. (2001) The expression of functional LPS receptor proteins CD14 and toll-like receptor 4 in human corneal cells. Invest Ophthalmol Vis Sci 42: 2867-2877.

40. Dart JK, Radford CF, Minassian D, Verma S, Stapleton F (2008) Risk factors for microbial keratitis with contemporary contact lenses: a case-control study. Ophthalmology 115: 1647-165, 1654.

41. Srinivasan $M$, Mascarenhas $J$, Rajaraman $R$, Ravindran $M$, Lalitha $P$, et al. (2012) Corticosteroids for bacterial keratitis: the steroids for corneal ulcers tria (SCUT). Arch Ophthalmol 130: 143-150.

42. McClellan S, Jiang X, Barrett R, Hazlett LD (2015) High-mobility group box 1 : a novel target for treatment of Pseudomonas aeruginosa keratitis. J Immunol 194: 1776-1787

43. Serhan CN (2014) Pro-resolving lipid mediators are leads for resolution physiology. Nature 510: 92-101.

44. Lee JE, Sun Y, Gjorstrup P, Pearlman E (2015) Inhibition of Corneal Inflammation by the Resolvin E1. Invest Ophthalmol Vis Sci 56: 2728-2736.

45. Iseli HP, Thiel MA, Hafezi F, Kampmeier J, Seiler T (2008) Ultraviolet A riboflavin corneal cross-linking for infectious keratitis associated with corneal melts. Cornea 27: 590-594.

46. Zhu H, Kumar A, Ozkan J, Bandara R, Ding A, et al. (2008) Fimbrolide-coated antimicrobial lenses: their in vitro and in vivo effects. Optom Vis Sci 85: 292300 